\title{
ST3Gal III modulates breast cancer cell adhesion and invasion by altering the expression of invasion-related molecules
}

\author{
HONG-XIA CUI ${ }^{1}$, HONGLAN WANG ${ }^{1}$, YUCHUN WANG ${ }^{1}$, JUAN SONG $^{1}$, \\ HUA TIAN $^{1}$, CHUNHUI XIA ${ }^{1}$ and YETONG SHEN ${ }^{2}$ \\ ${ }^{1}$ Department of Pharmacology, Qiqihar Medical University, Qiqihar, Heilongjiang 161042; \\ ${ }^{2}$ The First Affiliated Hospital, Qiqihar Medical University, Qiqihar, Heilongjiang 161006, P.R. China
}

Received April 25, 2016; Accepted June 30, 2016

DOI: $10.3892 /$ or.2016.5180

\begin{abstract}
Changes in the carbohydrate structure on the surface of tumor cells is an important feature of cancer metastasis. The specific role of sialic acids in the glycoconjugate terminal has not yet been clearly elucidated in these processes. Previously, we reported that $\alpha 2,3$-sialic acid residues in breast cancer are associated with metastatic potential. The $\alpha 2,3-$ sialyltransferase ST3Gal III, which adds $\alpha 2,3$-sialic acids to glycoproteins, is overexpressed in various tumors, and enzyme activity is correlated with tumor metastasis, yet its mechanistic role has not been fully evaluated. In the present study, we aimed to investigate the influence of ST3Gal III on key steps in the process of breast cancer metastasis. ST3Gal III-overexpressing and ST3Gal III-silenced breast cancer MDA-MB-231 cell lines were generated. They showed an increase or decrease in the tumor-associated antigen sialyl-Lewis X (SLeX). The E-selectin binding capacity of the transfectants was proportional to cell surface SLeX levels. Cell migration and invasion were positively correlated with ST3Gal III levels. Moreover, ST3Gal III expression modulated the protein expression of invasion-related molecules, including $\beta_{1}$ integrin, matrix metalloproteinase (MMP)-2, MMP-9 and cyclooxygenase-2, which may account for the mechanism involved in the effects of ST3Gal III on breast cancer invasiveness. In conclusion, our findings in these novel models of ST3Gal III expression revealed a critical requirement for ST3Gal III in several steps of breast cancer metastasis. ST3Gal III modulates breast cancer cell adhesion and invasion by altering the expression of invasion-related molecules. This study provides novel insights into the mechanisms underlying metastasis and suggests a new target for the effective drug treatment of breast cancer metastasis.
\end{abstract}

Correspondence to: Dr Hong-Xia Cui, Department of Pharmacology, Qiqihar Medical University, 333 Bukui Street, Jianhua, Qiqihar, Heilongjiang 161042, P.R. China

E-mail: xutianfang@sohu.com

Key words: $\alpha 2,3$-sialyltransferase ST3Gal III, human breast cancer, adhesion, migration, invasion-related molecules

\section{Introduction}

Changes in the carbohydrate structure on the surface of tumor cells are an important feature of cancer metastasis. Sialic acid, which is ubiquitous at terminal positions of glycoconjugates and bears net charge, has attracted the increased attention of researchers (1). However, the role of sialic acids in the processes involved in cancer metastasis has not been clarified. Previously we reported that $\alpha 2,3$-sialic acid residues in breast cancer are associated with metastatic potential. $\alpha 2,3$-sialyltransferase ST3Gal III transfers sialic acid with $\alpha 2,3$-linkage to Gal residues located on either Galb1-4GlcNAc or Galb1-3GlcNAc structures (2).

Changes in ST3Gal III expression have been reported in several types of tumors. In breast cancer, high expression of ST3Gal III was found to be positively correlated with the number of axillary lymph nodes and reduced patient overall survival $(3,4)$. In extrahepatic bile duct carcinoma, ST3Gal III levels are correlated with metastasis and poor prognosis (5). In squamous cell carcinoma of the cervix, ST3Gal III expression levels were obviously increased in patients with lymph node metastasis when compared to levels in patients without metastases $(6,7)$. In colon cancer, ST3Gal III expression was increased in carcinoma specimens compared with that in non-malignant colorectal mucosa (8). In gastric cancer, high levels of ST3Gal III were found to be correlated with tumor recurrence (9).

Although ST3Gal III expression correlates with tumor progression in various types of carcinomas (10), the mechanism of ST3Gal III in the process of metastasis has not been fully clarified. ST3Gal III is involved in the biosynthesis of sialyl-Lewis antigens, which can bind to E-selectin expressed on activiated endothelial cells and mediate hematogenous metastasis (11-13). In the present study, we investigated the specific influence of ST3Gal III on key steps in breast cancer progression, such as adhesion, migration and metastasis. Toward this aim, we chose two breast cancer cell lines MDA-MB-231 and Bcap37, with different ST3Gal III expression, and we generated an ST3Gal III-overexpressing cell line and downregulated ST3Gal III expression in a cell line by lentiviral transfection. ST3Gal III expression was proportional to sialyl-Lewis X (SLeX) levels and modulated the abilities of binding to E-selectin, cell migration and 
cell invasion. Furthermore, changes in expression of invasion-related molecules, including $\beta_{1}$ integrin, matrix metalloproteinase (MMP)-2, MMP-9 and cyclooxygenase-2 (COX-2) were found in both cell models, which could explain the effect of ST3Gal III on breast cancer cell adhesion and invasion.

In conclusion, our findings in these novel models of ST3Gal III expression revealed a critical requirement for ST3Gal III in several steps of breast cancer metastasis. ST3Gal III modulated breast cancer cell adhesion and invasion by altering the expression of invasion-related molecules. This study provides new insights into the mechanisms underlying metastasis and presents a new target for the effective drug treatment of breast cancer metastasis.

\section{Materials and methods}

Stable transfection. The construct human $\alpha 2,3$-sialyltransferase (human ST3Gal III) and short hairpin RNAs (shRNAs) targeting ST3Gal III and cloned into a lentivirus were synthesized by Suzhou GenePharma (Suzhou, China). The nucleotide sequence was confirmed by DNA sequencing. The encoding ST3Gal III lentivirus vector, four shRNA sequences targeting the ST3Gal III lentivirus vector and the empty lentivirus vector were transfected into human breast cancer cell lines MDA-MB-231 and Bcap37 (purchased from the Chinese Academy of Sciences Cell Bank, Shanghai, China) with different ST3Gal III expression. Stable transfected cells were selected with $2 \mu \mathrm{g} / \mathrm{ml}$ puromycin (InvivoGen, San Diego, CA, USA), and resistant clones were further confirmed by real-time PCR and western blot analysis.

Culture conditions for the transfected cells. The stable ST3Gal III-transfected MDA-MB-231 cells (ST3), shRNA targeting ST3Gal III-transfected MDA-MB-231 cells (shRNA-1, shRNA-2, shRNA-3 and shRNA-4, respectively), mock-transfected cells (M) and parental cells (P) were used in this study. Cells were cultured in L15 medium $\left(\mathrm{Gibco}^{\mathrm{TM}}\right.$ Invitrogen Corp., Carslbad, CA, USA) containing 10\% fetal bovine serum (FBS), $100 \mathrm{U} / \mathrm{ml}$ penicillin and $100 \mathrm{mg} / \mathrm{ml}$ streptomycin. Stable transfectants were supplemented with $2 \mu \mathrm{g} / \mathrm{ml}$ puromycin. Cells were fed every 3 days at $37^{\circ} \mathrm{C}$ in a humid atmosphere of $5 \% \mathrm{CO}_{2}$ and harvested by $0.25 \%$ trypsin. Cell growth and morphology were daily assessed under a field microscope.

ST3Gal III expression by real-time quantitative PCR. Total cellular RNA was extracted using TRIzol reagent (Invitrogen). Quantitative real-time PCR was performed using SYBR Premix Ex Taq (Takara Biotechnology Co., Ltd., Dalian, China) according to the manufacturer's instructions. For real-time PCR, we used ST3Gal III gene forward, 5'-AGAGA AGGACGGTGCCAAG-3' and reverse, 5'-CTGAATGAGGCT GAGTGCTG-3'; $\beta$-actin gene forward, 5'-GTGGACATCC GCA AAGAC-3' and reverse, 5'-GAAAGGGTGTAACGCA ACT-3'. The following standard thermal profile was used for all PCRs: $95^{\circ} \mathrm{C}$ for $10 \mathrm{~min} ; 40$ cycles of $95^{\circ} \mathrm{C}$ for $10 \mathrm{sec}$ and $60^{\circ} \mathrm{C}$ for $31 \mathrm{sec}$. For each sample dilution, logarithmic increase in fluorescence signal (DRn) was obtained. DRn threshold was set at 0.02 to obtain the corresponding $\mathrm{Ct}$ (threshold cycle) values. The endogenous housekeeping gene $\beta$-actin was used to normalize the results. Results are expressed as mean $\pm \mathrm{SD}$.

Flow cytometric analysis. Detection of oligosaccharide epitopes on the cell surface was performed by indirect fluorescence. Cells $\left(5 \times 10^{5}\right)$ were incubated with anti-SLeX monoclonal antibodies (MAbs) (Calbiochem, EMD Chemicals Inc., San Diego, CA, USA) at $4^{\circ} \mathrm{C}$ for $30 \mathrm{~min}$. After washing, the cells were incubated with the secondary antibody Alexa Fluor 594 goat anti-mouse IgG (Invitrogen Life Technologies, Frederick, MD, USA). Antibodies were diluted in phosphate-buffered saline (PBS) containing 1\% bovine serum albumin (BSA). Mean fluorescence intensity (MFI) was calculated using FACSCalibur (BD Biosciences, Franklin Lakes, NJ, USA). For each sample three independent assays were performed.

E-selectin binding assay. Adhesion of breast cancer cells to recombinant human E-selectin (rh-E-selectin) was performed. 96-well plates were coated with rh-E-selectin (R\&D Systems, Inc., Minneapolis, MN, USA) for $30 \mathrm{~min}$. The plates were blocked and $1 \times 10^{5}$ viable M, P, ST3 or shRNA-2, shRNA-4 cells, were added and incubated at $37^{\circ} \mathrm{C}$ for $1 \mathrm{~h}$. In the selected experiments, the cells were previously incubated with antiSLeX MAbs for $30 \mathrm{~min}$ at $4^{\circ} \mathrm{C}$. After washes, the adherent cells were fixed with methanol for $30 \mathrm{~min}$. After drying, the fixed cells were stained with $0.1 \%$ crystal violet for $30 \mathrm{~min}$, and then washed and dissolved in 10\% acetic acid. The optical densities of each well were measured by an ELISA reader at $570 \mathrm{~nm}$. All the experiments were performed in quintuplicate, and three independent assays were carried out. Results are expressed as the mean $\pm \mathrm{SD}$.

Tumor cell adhesion assay to HUVECs. Human umbilical vein endothelial cells (HUVECs) (purchased from the Chinese Academy of Sciences Cell Bank) were incubated for $12 \mathrm{~h}$ with $10 \mathrm{ng} / \mathrm{ml}$ recombinant interleukin IL-1 $\beta$ (R\&D Systems, Inc.). Cells were stained with calcein (Invitrogen Life Technologies) in L15 solution. After washing, the cells were resuspended and added to a 96-well plate and cultured with HUVECs. The plates were incubated at $37^{\circ} \mathrm{C}$ for $30 \mathrm{~min}$, and then washed three times and the number of adhesive cells was counted under a fluorescence microscope. Results are expressed as the mean \pm SD. Each experiment was performed in triplicate wells and three independents assays were carried out.

Cell migration and invasion assays. Tumor cell migration and invasion were assessed using Transwell chamber (Corning Costar, Inc., Corning, NY, USA). Briefly, Matrigel was used to coat the upper chamber for the invasion assay (not for the migration assay). Cells $\left(1 \times 10^{5}\right)$ were added to the upper chamber in serum-free medium, and the lower compartment was filled with media supplemented with $10 \%$ FBS. After incubation at $37^{\circ} \mathrm{C}$ for $24 \mathrm{~h}$, the non-migrating cells on the upper surface of the filter were removed with cotton swabs, and the migrating cells on the lower surface were fixed and stained with $0.1 \%$ crystal violet for $30 \mathrm{~min}$, then photographed under a fluorescence microscope and incorporated dye was dissolved in $10 \%$ acetic acid. The optical densities of each well were measured by ELISA reader at $570 \mathrm{~nm}$. Experiments were performed in triplicate. 


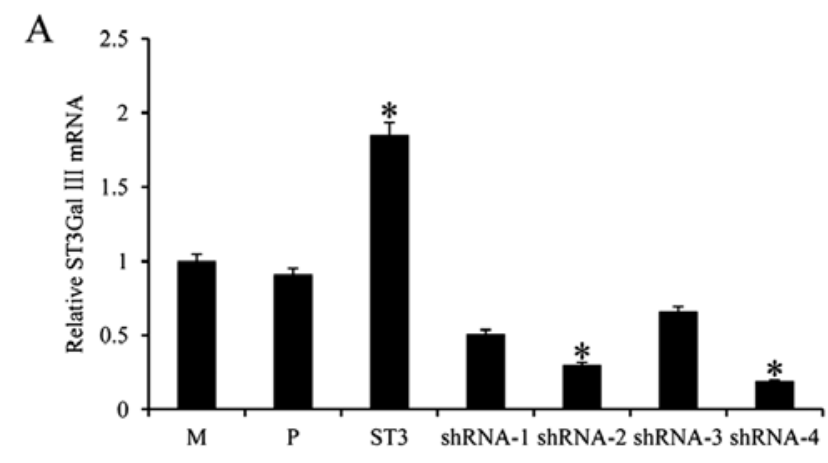

B
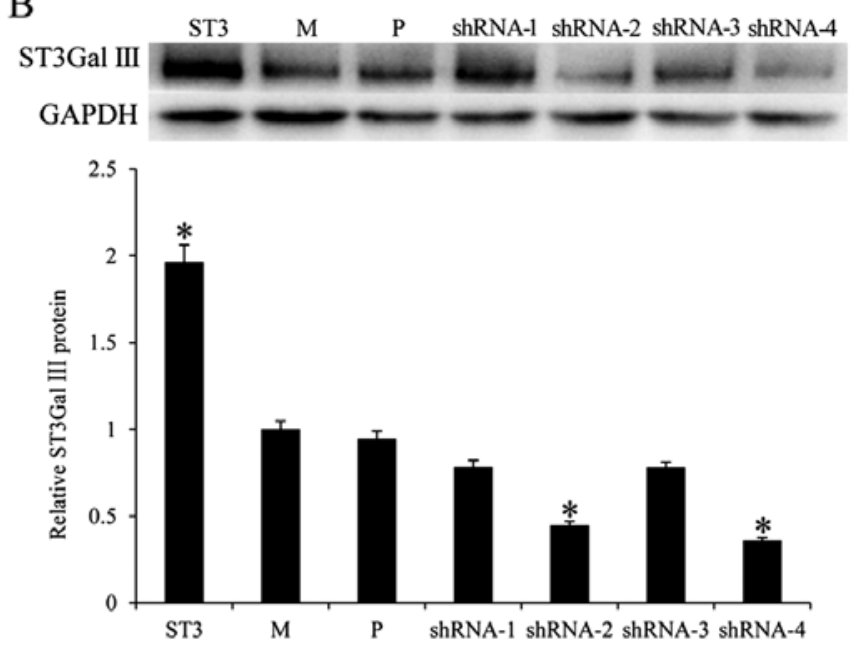

Figure 1. ST3Gal III expression in MDA-MB-231 cells. (A) The mRNA expression of ST3Gal III was determined by quantitative PCR. (B) The protein expression of ST3Gal III was examined by western blot analysis. M, MDA-MB-231 mock cells; P, MDA-MB-231 parental cells; ST3, MDAMB-231 cells transfected with the ST3Gal III gene; shRNA, MDA-MB-231 cells transfected with the shRNA sequences targeting ST3Gal III. For one experiment, three assays were carried out and only one set of gels is shown. The density of the band (normalized to GAPDH) is shown as mean \pm SD $(\mathrm{n}=3) .{ }^{*} \mathrm{P}<0.05$, compared to $\mathrm{P}$.

Western blot analysis. Total cellular proteins were extracted. Equivalent amounts of cellular protein were electrophoresed on $10 \%$ SDS-PAGE gel and transfered to nitrocellullose membranes (Millipore Corp., Billerica, MA, USA) and blocked in 5\% non-fat milk in TBST for $1 \mathrm{~h}$ at room temperature. The cells were incubated with primary antibodies to human ST3Gal III (Abcam, Cambridge, MA, USA), MMP-9, MMP-2 (Cell Signaling Technology, Inc., Beverly, MA, USA), $\beta_{1}$ integrin (Chemicon, Temecula, CA, USA), COX-2 and GAPDH (both from Santa Cruz Biotechnology, Inc., Santa Cruz, CA, USA) in $5 \%$ non-fat milk at $4^{\circ} \mathrm{C}$ overnight. The membranes were washed with TBST and incubated with horseradish peroxidase-conjugated secondary antibody in 5\% non-fat milk for $1 \mathrm{~h}$ at room temperature. Immune complexes were detected by enhanced chemoluminescence techniques (Amersham Life Science, Piscataway, NJ, USA). Band densities were quantified by using BandScan software.

Statistical analysis. The data are expressed as the mean $\pm \mathrm{SD}$ and were analyzed by SPSS 17.0 statistical software to evaluate the statistical difference. $\mathrm{P}<0.05$ was considered to indicate a statistically significant result. One-way ANOVA analysis was used to evaluate all quantitative data.

\section{Results}

Stable expression of ST3Gal III in MDA-MB-231 variant cells. To explore the role of ST3Gal III in breast cancer metastasis, the human ST3Gal III gene, and shRNA targeting ST3Gal III were cloned to lentiviral vectors. Breast cancer cells, MDA-MB-231 and Bcap37, were transfected with the lentiviral vector encoding the human ST3Gal III gene and MDA-MB-231 cells were transfected with ST3Gal III shRNAs. Parental cells were concomitantly transfected with the empty lentiviral vector. Stable cell clones were selected by puromycin and the mRNA levels of ST3Gal III were quantified by real-time PCR (Fig. 1A). ST3Gal III mRNA expression in the stable ST3Gal III-transfected MDA-MB-231 cells (ST3 cells) was 1.846 -fold higher $(\mathrm{P}<0.05)$ than that in the parental $(\mathrm{P})$ cells. For the Bcap37 cell model, there was no significant difference between the Bcap37 ST3 and P cells (data not shown). In contrast, ST3Gal III mRNA expression was 3.33-fold lower $(\mathrm{P}<0.05)$ in the shRNA-2-transfected cells and 5.26-fold lower $(\mathrm{P}<0.05)$ in the shRNA-4-transfected cells than that in the $\mathrm{P}$ cells. The protein expression of ST3Gal III was examined by western blot analysis. As expected, protein expression of ST3Gal III was increased in the ST3 cells $(\mathrm{P}<0.05)$ and decreased in the shRNA-2 and shRNA-4 transfected cells $(\mathrm{P}<0.05)$ when compared with the P cells (Fig. 1B). For shRNA-1 and shRNA-3 transfected cells, there was no significant difference in ST3Gal III mRNA and protein expression. Thus, ST3Gal III-overexpressing ST3 cells (for the MDA-MB-231 model) and shRNA-2 and shRNA-4 transfected cells with downregulated ST3Gal III expression were selected for further studies. As controls, mock-transfected clones (MDA-MB-231), $\mathrm{M}$ and parental cell clones, $\mathrm{P}$ were used.

ST3Gal III modulates de novo expression of SLeX. The synthesis of SLeX structures was catalyzed by ST3Gal III. The levels of SLeX could resort to ST3Gal III enzyme activities. Carbohydrate structure on the cell surface was studied by flow cytometry using specific MAbs against SLeX. The results revealed that ST3 clones displayed a large increase in SLeX expression and a significant decrease in SLeX expression was observed in the shRNA-2 and shRNA-4 transfected cells, compared to the controls $(\mathrm{P}<0.05)$ (Fig. 2).

Breast cancer cell binding to rh-E-selectin is correlated with SLeX levels. SLeX present on the tumor cell surface can mediate tumor hematogenous spread by binding to E-selectin expressed on activated endothelial cells. rh-E-selectin binding assays were used to analyze whether the different levels of SLeX expression could regulate changes in the adhesion to rh-Eselectin. As shown in Fig. 3, both cell clones showed different adhesion patterns. The capacity for adhesion to rh-E-selectin was enhanced in the ST3 cells with higher SLeX expression compared with that in the $\mathrm{M}$ and $\mathrm{P}$ cells $(\mathrm{P}<0.05)$. Obviously, lower adhesion to rh-E-selectin was noted in the shRNA-2 and shRNA-4 transfected cells than that noted in the M and $\mathrm{P}$ cells $(\mathrm{P}<0.05)$, which was consistent with the lower expression level 
A

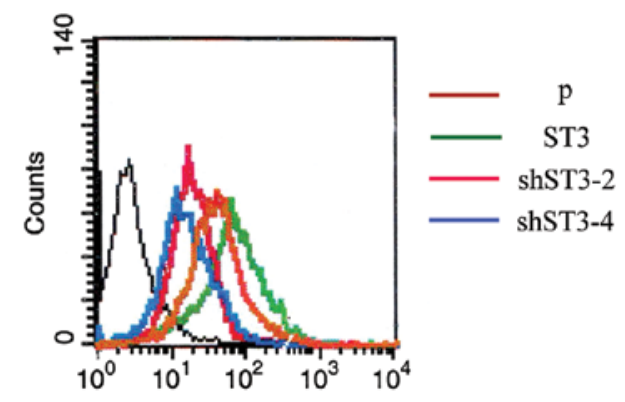

B

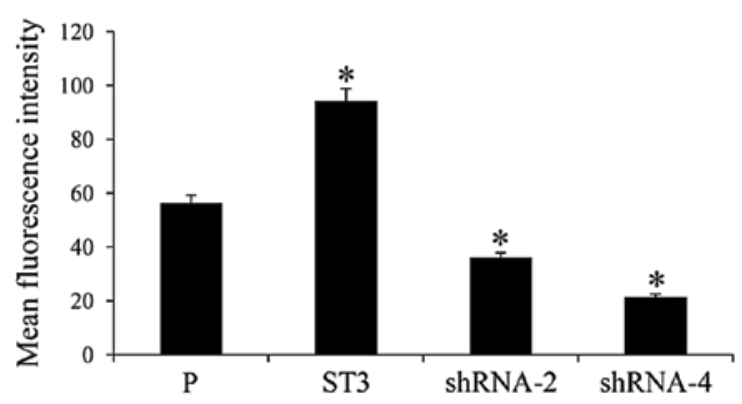

Figure 2. Flow cytometric analysis of sialyl-Lewis X (SLeX) structures on the cell surface of breast cancer cells. Cells were incubated with anti-SLeX monoclonal antibody (MAb) at $4^{\circ} \mathrm{C}$ for $1 \mathrm{~h}$. (A) The fluorescence intensities of the labeled cells were determined using flow cytometry. (B) SLeX expression was markedly increased in the STS clone, and SLeX expression was markedly decreased in the shRNA-2 and shRNA-4 transfected clones compared with that in the parental cells $(\mathrm{P})$. Values of mean fluorescence intensity (MFI) are shown as mean $\pm \mathrm{SD}(\mathrm{n}=3)$. ${ }^{*} \mathrm{P}<0.05$, compared to $\mathrm{P}$. $\mathrm{P}$, MDA-MB-231 parental cells; ST3, MDA-MB-231 cells transfected with the ST3Gal III gene; shRNA-2 and shRNA-4, ST3Gal III-silenced cells.

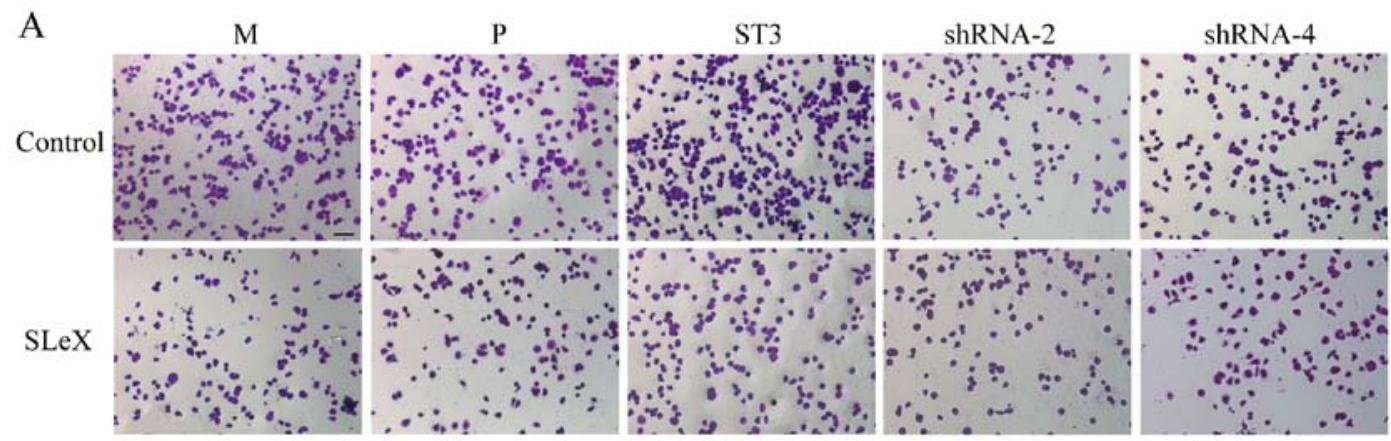

B

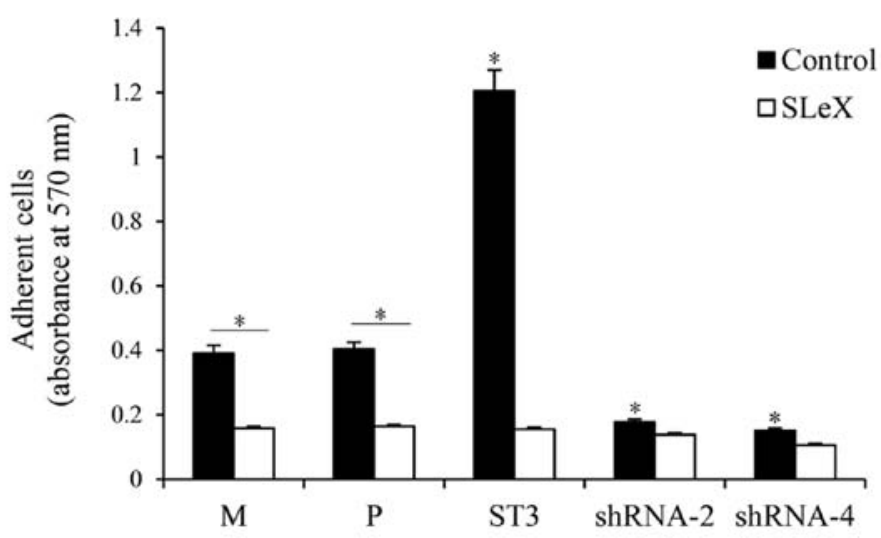

Figure 3. Assay for detection of the binding to recombinant human E-selectin (rh-E-selectin). Cells previously incubated with anti-sialyl-Lewis X (SLeX) monoclonal antibody (MAb) or not, were added to 96-well microplates coated with rh-E-selectin, and then adherent cells were dyed and dissolved in 10\% acetic acid. The optical densities of each well were measured by ELISA reader at $570 \mathrm{~nm}$. (A) The images show crystal violet-stained breast cancer cells binding to rh-E-selectin (magnification, $\mathrm{x} 100$ ). Scale bar, $100 \mu \mathrm{m}$. (B) The optical densities of adherent cells are shown as mean \pm SD ( $\mathrm{n}=3$ ). Control groups were incubated without anti-SLeX-MAb; SLeX groups were incubated with anti-SLeX-MAb. * $\mathrm{P}<0.05$, compared to P. M, MDA-MB-231 mock cells; P, MDAMB-231 parental cells; ST3, MDA-MB-231 cells transfected with the ST3Gal III gene; shRNA-2 and shRNA-4, ST3Gal III-silenced cells.

of SLeX. To further confirm that SLeX expression induced by ST3Gal III caused regulation of rh-E-selectin binding, we preincubated both cell clones with anti-SLeX MAb. Cell adhesion to E-selectin was significantly decreased in the ST3 cells $(\mathrm{P}<0.05)$, whereas shRNA cells, which had lower SLeX expression levels, did not show a significant change in adhesion capacity to rh-E-selectin. Moreover, there was no different adhesion ability among the groups after incubation. These results demonstrated that the E-selectin binding capacity of the transfectants was proportional to cell surface SLeX levels.
ST3Gal III expression mediates breast cancer cell adhesion to HUVECs. We next determined the adhesion of the MDA-MB231 cells with different ST3Gal III expression levels to HUVECs pretreated with IL-1 $\beta$. ST3 cells with high SLeX expression had a higher adhesion to HUVECs, and shRNA-2, shRNA-4 cells showed significantly decreased adhesion to HUVECs, compared with that observed in the $\mathrm{M}$ and $\mathrm{P}$ cells $(\mathrm{P}<0.05)$ (Fig. 4). These data showed that ST3Gal III and SLeX play an important role in mediating breast cancer adhesion to HUVECs. 

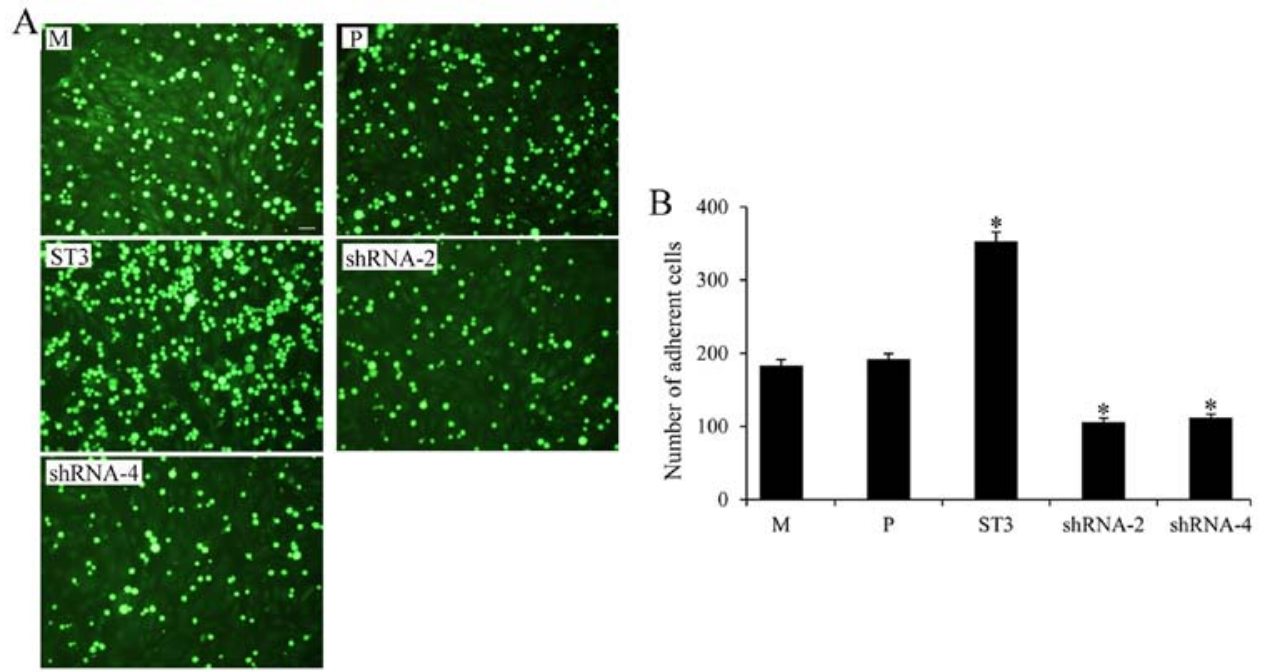

Figure 4. Assay of tumor cell adhesion to human umbilical vein endothelial cells (HUVECs). MDA-MB-231 variant cells were labelled with calcein and added to IL-1 $\beta$-stimulated HUVECs. (A) The images show the calcein-stained breast cancer cell adhesion to HUVECs (magnification, $\mathrm{x} 40$ ). Scale bar, $200 \mu \mathrm{m}$. (B) The numbers of adherent cells are shown as mean $\pm \mathrm{SD}(\mathrm{n}=3)$. ${ }^{*} \mathrm{P}<0.05$, compared to P. M, MDA-MB-231 mock cells; P, MDA-MB-231 parental cells; ST3, MDA-MB-231 cells transfected with the ST3Gal III gene; shRNA-2 and shRNA-4, ST3Gal III-silenced cells.

A

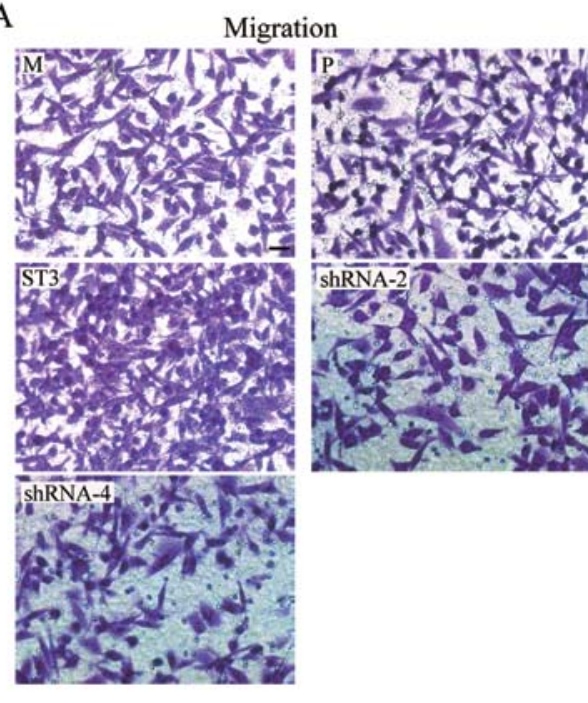

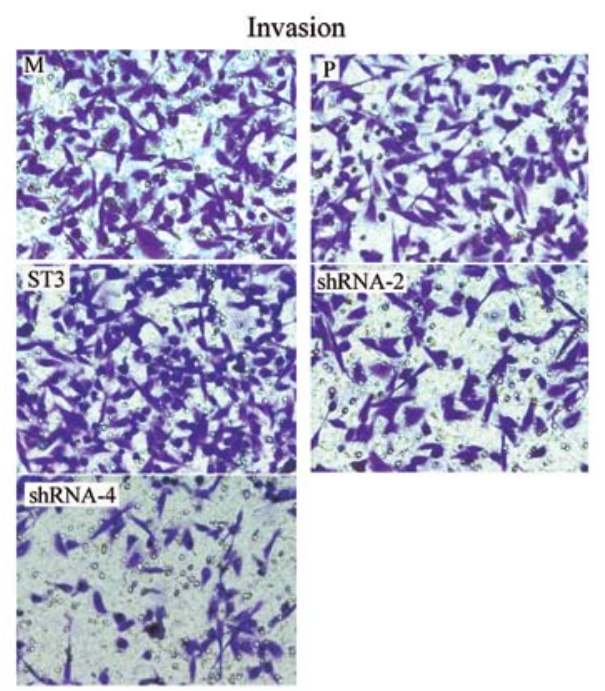

$\mathrm{C}$

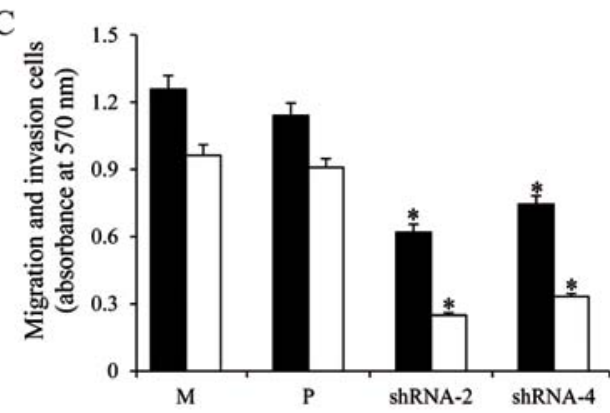

Figure 5. Cell migration and invasion were examined using Transwell chambers. (A) Images show crystal violet-stained breast cancer cells migrating through Transwell chambers (magnification, x200). Scale bar, $50 \mu \mathrm{m}$. (B) ST3 cells displayed enhanced migration and invasion potential compared with M and P. (C) shRNA-2 and shRNA-4 cells showed a significant reduction in migration and invasion compared with $\mathrm{M}$ and $\mathrm{P}$. The optical densities of migration and invasion cells are shown as mean \pm SD $(n=3)$. $P<0.05$, compared to P. M, MDA-MB-231 mock cells; P, MDA-MB-231 parental cells; ST3, MDA-MB-231 cells transfected with the ST3Gal III gene; shRNA-2 and shRNA-4, ST3Gal III-silenced cells.

ST3Gal III expression levels modulate cell migration and invasion. To further investigate the relationship between ST3Gal III expression and the acquisition of a more invasive cell phenotype, we evaluated cell migration and invasion using Transwell chamber. As shown in Fig. 5, both cell clones showed different migratory capabilities; the ST3Gal III-overexpressing 
A
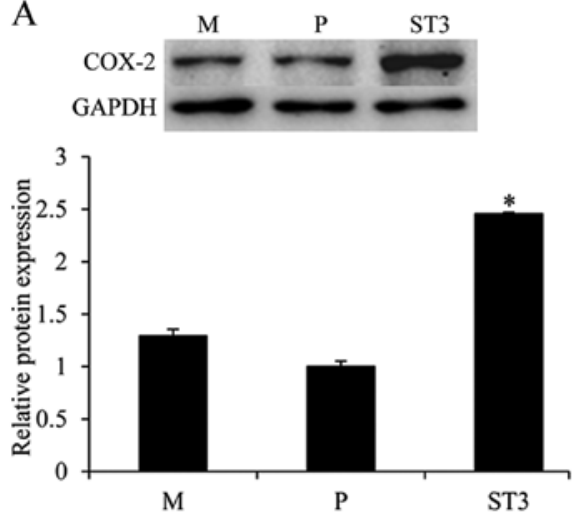

$\mathrm{C}_{\beta_{1} \text { integrin }}$ GAPDH

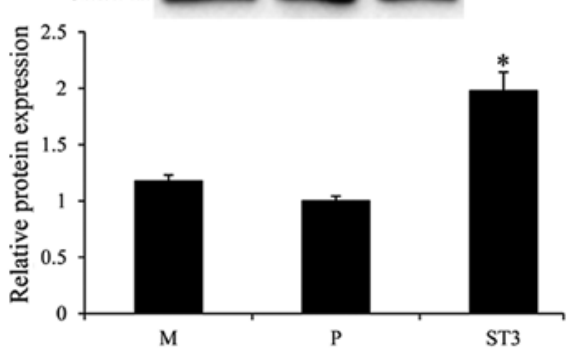

E
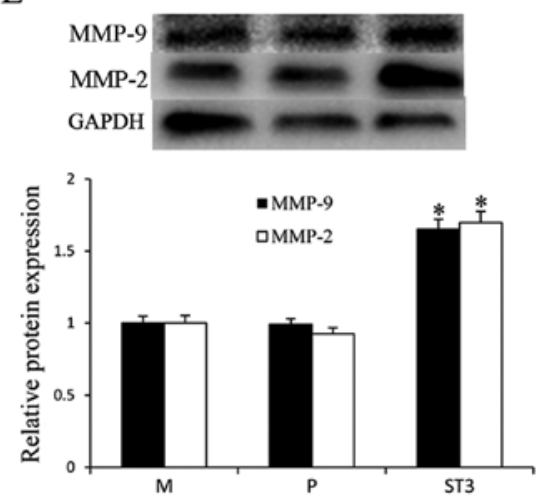

B
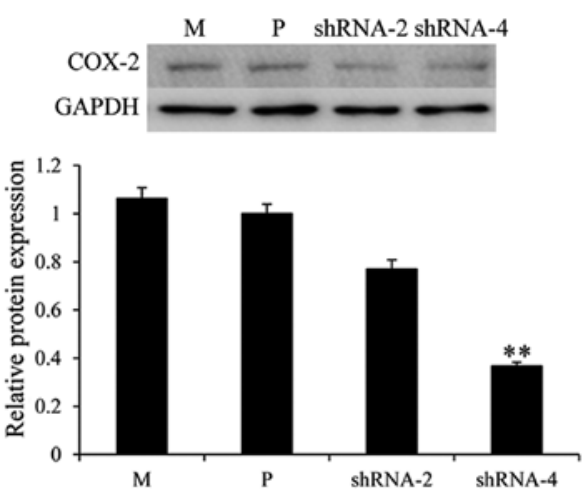

$\mathrm{D}$

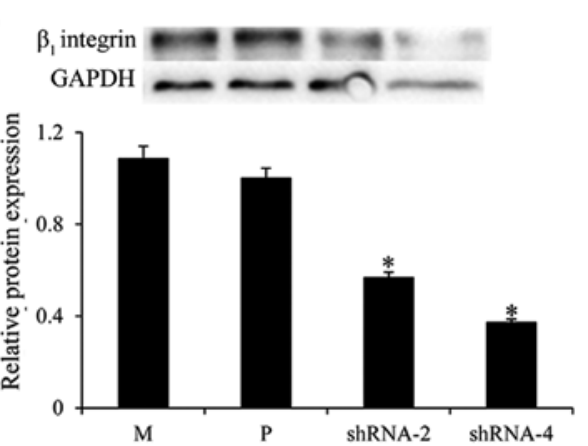

$\mathrm{F}$
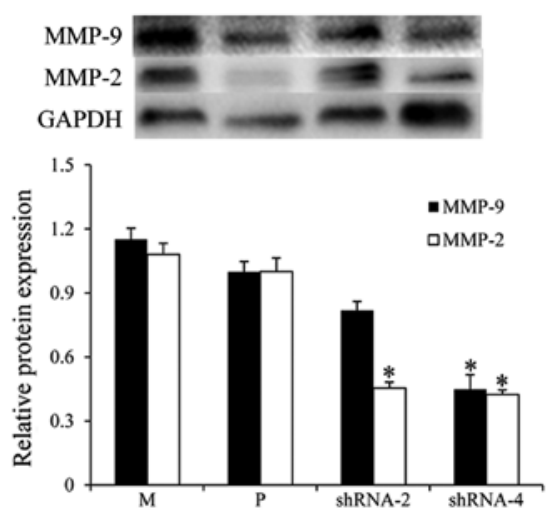

Figure 6. ST3Gal III induces the expression of cyclooxygenase-2 (COX-2), $\beta_{1}$ integrin, matrix metalloproteinase (MMP)-2 and MMP-9. The expression of invasion-related molecules, COX-2, $\beta_{1}$ integrin, MMP-2 and MMP-9 was determined via western blot analysis. (A, C and E) ST3 cells displayed increased COX-2, $\beta_{1}$ integrin, MMP-2 and MMP-9 expression, whereas (B, D and F) shRNA-2 and shRNA-4 cells showed a significant reduction in expression levels. The density of the bands (normalized to GAPDH) are shown as mean $\pm \mathrm{SD}(\mathrm{n}=3) .{ }^{*} \mathrm{P}<0.05,{ }^{* *} \mathrm{P}<0.01$ compared to P. M, MDA-MB-231 mock cells; $\mathrm{P}, \mathrm{MDA}-$ MB-231 parental cells; ST3, MDA-MB-231 cells transfected with the ST3Gal III gene; shRNA-2 and shRNA-4, ST3Gal III-silenced cells.

clone ST3 exhibited higher migratory capability $(\mathrm{P}<0.05)$, and the ST3Gal III-silenced clones shRNA-2 and shRNA-4 exhibited decreased migration compared with the $\mathrm{M}$ and $\mathrm{P}$ cells $(\mathrm{P}<0.05)$. The results demonstrated a positive correlation between ST3Gal III levels and invasive capabilities.

ST3GalIII induces the expression of COX-2, $\beta_{1}$ integrin, MMP-2 and MMP-9. To further investigate the molecular mechanism of ST3Gal III in modulating cell migration and invasion, we examined the protein expression of invasion-related molecules, COX-2, $\beta_{1}$ integrin, MMP-2 and MMP-9 using western blot analysis. The results showed that ST3Gal III-overexpressing ST3 cells had increased COX-2 (Fig. 6A), $\beta_{1}$ integrin (Fig. 6C), MMP-2 and MMP-9 expression $(\mathrm{P}<0.05)$ (Fig. 6E). Moreover, the expression of COX-2 (Fig. 6B), $\beta_{1}$ integrin (Fig. 6D), MMP-2 and MMP-9 (Fig. 6F) were downregulated in the
shRNA-2 and shRNA-4 cells $(\mathrm{P}<0.05)$. These results showed that ST3Gal III induced the expression of COX-2, $\beta_{1}$ integrin, MMP-2 and MMP-9.

\section{Discussion}

Our previous study showed that $\alpha 2,3$-sialic acid residues in breast cancer are associated with metastatic potential (14). We used $\alpha 2,3$-sialyltransferase ST3Gal III-overexpressing clone ST3 and shRNA-targeted ST3Gal III clone shRNA cells, and extended our study to investigate the mechanistic role of ST3Gal III in the process of metastasis, such as adhesion, migration and invasion in MDA-MB-231 breast cancer cell lines. The results showed that ST3Gal III expression increased sialylation structure SLeX expression. ST3Gal III regulated the abilities of breast cancer cell binding to rh-E-selectin and 
IL1- $\beta$-pretreated HUVECs, via SLeX-E-selectin interaction. In addition, a positive correlation between invasive capacity and ST3Gal III expression was found. Our finding showed that ST3Gal III expression plays a critical role in several steps of the process of breast cancer metastasis.

The molecular mechanisms regulating breast cancer metastasis are still poorly understood. Sialic acids overexpressed in some tumors have the potential to modulate interactions between molecules and cells. One crucial molecule is SLeX with $\alpha 2,3$-sialic acid residues, which involves the attachment of tumor cells to activated endothelial cells. SLeX has been reported to participate in the processes of extravasation by interacting with E-selectin $(15,16)$. Several studies have reported that SLeX expression is directly correlated with the binding to endothelial cells via E-selectin-SLeX interaction in colon cancer (17), H7721 hepatocarcinoma (18) and lung adenocarcinoma cells (19). In agreement with these data, our results showed that the ST3Gal III-overexpressing ST3 cells exhibited enhanced adherence to rh-E-selectin and the ST3Gal III-silenced shRNA cells had reduced adhesion ability. Moreover, when studying the adhesion of breast cancer cells to IL-1 $\beta$-stimulated HUVECs, similar results further demonstrated that ST3Gal III and SLeX levels were correlated with E-selectin expressed on HUVECs. When we pretreated ST3 cells with anti-SLeX, the enhanced adhesion was obviously decreased, whereas shRNA cells with low SLeX levels had no significant change. After incubation, there was no difference among the groups, which could account for breast cancer cells binding to stimulated HUVECs mediated by SLeX.

Cell migration and invasion are multistep processes that play pivotal role in cancer metastasis. To our knowledge, $\alpha 2,3$-sialic acid residues are associated with the migration processes in cancer. When $\alpha 2,3$-sialic acid levels on the tumor cell surface were increased, cell migration was significantly enhanced (20). We aimed to ascertain whether ST3Gal III expression and the subsequent changes in $\alpha 2,3$-sialic acid levels have a role in invasion in MDA-MB-231 breast cancer cells. ST3Gal III-overexpressing ST3 cells exhibited high migration and invasion abilities, whereas ST3Gal III-silenced shRNA cells demonstrated lower invasion capabilities when compared with the mock and parental cells, which was in agreement that $\alpha 2,3$-sialic acid expression resulted in a more invasive phenotype in vitro (21). In addition, both cell clones ST3 and shRNA displayed different amounts of SLeX expression, which consequently show a different invasive potential. These results reinforce the importance of $\alpha 2,3$-sialic acids in potentiating cell invasion and metastasis. In accordance, decreased $\alpha 2,3$-sialic acid levels in a cancer model resulted in suppression of invasion and metastasis in lung cancer (22) and hepatocarcinoma (23). Moreover, migration was inhibited after sialic acid residue was elimination (24). To the best of our knowledge, this is the first report concerning a positive correlation between cell migration and ST3Gal III expression levels in breast cancer cells.

Integrins as extracellular matrix (ECM) adhesion molecules, are involved in metastatic progression, such as the formation of invadopodia (25), the interaction of metastatic tumor cells with their surrounding ECM (26), and acquisition of invasive behavior (27). The levels of $\beta_{1}$ integrin are correlated with metastatic potential. The results described here suggest that the ST3Gal III gene regulates the levels of $\beta_{1}$ integrin expression. We found that $\beta_{1}$ integrin expression was constitutively increased in the ST3Gal III-overexpressing ST3 cells, while low expression was noted in the ST3Gal III-silenced shRNA cells, which could be the machanism involved in the influence on cell invasion abilities. Several studies have demonstrated that sialylation influences migration capability by modulating the integrin function $(28,29)$. Thus, we infer that ST3Gal III could alter the sialylation levels of $\beta_{1}$ integrin, modulating their metastatic potential. Nevertheless, further investigations are required to address this issue.

COX-2 is undetectable in most normal tissues but can be induced in many cancers. High COX-2 expression has been reported in colon (30), bladder (31), and breast (32) cancers, and is associated with cell motility and invasion (33), angiogenesis (34), and lymph node metastasis (35). Interestingly, all these cancers show increased expression of ST3Gal III. The relationship between ST3Gal III and COX-2 protein expression was investigated. The results found that the ST3Gal III gene could induce the expression of COX-2. COX-2 expression was increased in the ST3 cells with ST3Gal III overexpression, and downregulated in the ST3Gal III-silenced shRNA cells compared with the mock and parental cells, which could be one of the mechanism of the influence on cell invasion abilities mediated by ST3Gal III.

MMP-9 and MMP-2, are important isoforms in the MMP family, and have been reported to be overexpressed and degrade collagen and gelatin, which can aid cell migration and angiogenesis (36). Recently, it was shown that expression of MMP-9 has been associated with shorter survival rates in breast cancer patients (37). Additionally, the pulmonary metastasis ability of cancer cells was found to be reduced in MMP-2- or MMP-9deficient mice compared to wild-type mice (38) and cancer cell proliferation was suppressed in tumors obtained from MMP-9knockout mice (39). Our results found that ST3 cells with ST3Gal III overexpression had increased expression of MMP-2 and MMP-9, which was decreased in the ST3Gal III-silenced shRNA cells, which may have resulted in the breast cancer cell invasion abilities mediated by ST3Gal III.

Tumor invasion and metastasis are known to be the major causes of mortality in breast cancer patients. In the present study, we engineered stable transfectants of ST3Gal III-overexpressing clone and shRNA-targeted ST3Gal III clones to globally evaluate the role of ST3Gal III in key steps of the process of breast cancer metastasis. In the present study, we demonstrated that ST3Gal III and its downstream product SLeX, conferred to breast cancer cells high adhesion to E-selectin, high migration and invasion capacities, and regulated expression of invasionrelated molecules $\beta_{1}$ integrin, COX-2, MMP-9 and MMP-2. In addition to the role of ST3Gal III in these key steps, this enzyme has been reported to produce resistance to Taxol therapy in ovarian cancer cells (40). This study highlights the importance of ST3Gal III in cancer processes. A next step would be to further design inhibitors targeted ST3Gal III that may inhibit breast cancer metastasis.

\section{Acknowledgements}

This study was supported by the National Youth Science Foundation of China (no. 81302308), the Natural Science 
Foundation of Heilongjiang Province, China (H201353) and the Youth Leading Scholar Supporting Program in General Colleges and Universities of Heilongjiang, China (no. 1253G067).

\section{References}

1. Varki A, Cummings RD, Esko JD, Freeze HH, Stanley P, Bertozzi CR, Hart GW and Etzler ME (eds): Essentials of Glycobiology. 2nd edition. Cold Spring Harbor Laboratory Press, New York, NY, 2009.

2. Harduin-Lepers A, Vallejo-Ruiz V, Krzewinski-Recchi MA, Samyn-Petit B, Julien S and Delannoy P: The human sialyltransferase family. Biochimie 83: 727-737, 2001.

3. Recchi MA, Hebbar M, Hornez L, Harduin-Lepers A, Peyrat JP and Delannoy P: Multiplex reverse transcription polymerase chain reaction assessment of sialyltransferase expression in human breast cancer. Cancer Res 58: 4066-4070, 1998.

4. Hebbar M, Krzewinski-Recchi MA, Hornez L, Verdière A, Harduin-Lepers A, Bonneterre J, Delannoy P and Peyrat JP Prognostic value of tumoral sialyltransferase expression and circulating E-selectin concentrations in node-negative breast cancer patients. Int J Biol Markers 18: 116-122, 2003.

5. Jin XL, Zheng SS, Wang BS and Chen HL: Correlation of glycosyltransferases mRNA expression in extrahepatic bile duct carcinoma with clinical pathological characteristics. Hepatobiliary Pancreat Dis Int 3: 292-295, 2004

6. Wang PH, Li YF, Juang CM, Lee YR, Chao HT, Ng HT, Tsai YC and Yuan CC: Expression of sialyltransferase family members in cervix squamous cell carcinoma correlates with lymph node metastasis. Gynecol Oncol 86: 45-52, 2002.

7. Wang PH, Lee WL, Lee YR, Juang CM, Chen YJ, Chao HT, Tsai YC and Yuan CC: Enhanced expression of alpha 2,6-sialyltransferase ST6Gal I in cervical squamous cell carcinoma. Gynecol Oncol 89: 395-401, 2003.

8. Petretti T, Kemmner W, Schulze B and Schlag PM: Altered mRNA expression of glycosyltransferases in human colorectal carcinomas and liver metastases. Gut 46: 359-366, 2000.

9. Gretschel S, Haensch W, Schlag PM and Kemmner W: Clinical relevance of sialyltransferases ST6GAL-I and ST3GAL-III in gastric cancer. Oncology 65: 139-145, 2003.

10. Varki A: Glycan-based interactions involving vertebrate sialic-acid-recognizing proteins. Nature 446: 1023-1029, 2007.

11. Hosono J, Narita T, Kimura N, Sato M, Nakashio T, Kasai Y, Nonami T, Nakao A, Takagi H and Kannagi R: Involvement of adhesion molecules in metastasis of SW1990, human pancreatic cancer cells. J Surg Oncol 67: 77-84, 1998.

12. Mas E, Pasqualini E, Caillol N, El Battari A, Crotte C, Lombardo D and Sadoulet MO: Fucosyltransferase activities in human pancreatic tissue: Comparative study between cancer tissues and established tumoral cell lines. Glycobiology 8: 605-613, 1998.

13. Peracaula R, Tabarés G, López-Ferrer A, Brossmer R, de Bolós C and de Llorens R: Role of sialyltransferases involved in the biosynthesis of Lewis antigens in human pancreatic tumour cells. Glycoconj J 22: 135-144, 2005.

14. Cui H, Lin Y, Yue L, Zhao X and Liu J: Differential expression of the $\alpha 2,3$-sialic acid residues in breast cancer is associated with metastatic potential. Oncol Rep 25: 1365-1371, 2011

15. Kannagi R, Izawa M, Koike T, Miyazaki K and Kimura N: Carbohydrate-mediated cell adhesion in cancer metastasis and angiogenesis. Cancer Sci 95: 377-384, 2004.

16. Kobayashi H, Boelte KC and Lin PC: Endothelial cell adhesion molecules and cancer progression. Curr Med Chem 14: 377-386, 2007.

17. Matsushita Y, Kitajima S, Goto M, Tezuka Y, Sagara M, Imamura $\mathrm{H}$, Tanabe $\mathrm{G}$, Tanaka $\mathrm{S}$, Aikou $\mathrm{T}$ and Sato E: Selectins induced by interleukin-1beta on the human liver endothelial cells act as ligands for sialyl Lewis X-expressing human colon cancer cell metastasis. Cancer Lett 133: 151-160, 1998.

18. Wu SL, Ma J, Qi HL, Zhang Y, Zhang XY and Chen HL: Forskolin up-regulates metastasis-related phenotypes and molecules via protein kinase B, but not PI-3K, in H7721 human hepato-carcinoma cell line. Mol Cell Biochem 254: 193-202, 2003

19. Martín-Satué M, de Castellarnau C and Blanco J: Overexpression of alpha(1,3)-fucosyltransferase VII is sufficient for the acquisition of lung colonization phenotype in human lung adenocarcinoma HAL-24Luc cells. Br J Cancer 80: 1169-1174, 1999.
20. Bassagañas S, Pérez-Garay M and Peracaula R: Cell surface sialic acid modulates extracellular matrix adhesion and migration in pancreatic adenocarcinoma cells. Pancreas 43: 109-117, 2014.

21. Pérez-Garay M, Arteta B, Pagès L, de Llorens R, de Bolòs C, Vidal-Vanaclocha F and Peracaula R: alpha2,3-sialyltransferase ST3Gal III modulates pancreatic cancer cell motility and adhesion in vitro and enhances its metastatic potential in vivo. PLoS One 5: e12524, 2010.

22. Lalor PF, Edwards S, McNab G, Salmi M, Jalkanen S and Adams DH: Vascular adhesion protein-1 mediates adhesion and transmigration of lymphocytes on human hepatic endothelial cells. J Immunol 169: 983-992, 2002.

23. Liu F, Qi HL, Zhang Y, Zhang XY and Chen HL: Transfection of the c-erbB2/neu gene upregulates the expression of sialyl Lewis X, alpha1,3-fucosyltransferase VII, and metastatic potential in a human hepatocarcinoma cell line. Eur J Biochem 268: 3501-3512, 2001.

24. Zhang Y, Zhang XY, Liu F, Qi HL and Chen HL: The roles of terminal sugar residues of surface glycans in the metastatic potential of human hepatocarcinoma. J Cancer Res Clin Oncol 128: 617-620, 2002.

25. Beaty BT, Sharma VP, Bravo-Cordero JJ, Simpson MA, Eddy RJ, Koleske AJ and Condeelis J: $\beta 1$ integrin regulates Arg to promote invadopodial maturation and matrix degradation. Mol Biol Cell 24: 1661-1675, S1-S11, 2013.

26. Shibue T, Brooks MW and Weinberg RA: An integrin-linked machinery of cytoskeletal regulation that enables experimental tumor initiation and metastatic colonization. Cancer Cell 24: 481-498, 2013.

27. Nieto MA and Cano A: The epithelial-mesenchymal transition under control: Global programs to regulate epithelial plasticity. Semin Cancer Biol 22: 361-368, 2012.

28. Shaikh FM, Seales EC, Clem WC, Hennessy KM, Zhuo Y and Bellis SL: Tumor cell migration and invasion are regulated by expression of variant integrin glycoforms. Exp Cell Res 314: 2941-2950, 2008.

29. Seales EC, Jurado GA, Brunson BA, Wakefield JK, Frost AR and Bellis SL: Hypersialylation of beta1 integrins, observed in colon adenocarcinoma, may contribute to cancer progression by up-regulating cell motility. Cancer Res 65: 4645-4652, 2005.

30. Eberhart CE, Coffey RJ, Radhika A, Giardiello FM, Ferrenbach $S$ and DuBois RN: Up-regulation of cyclooxygenase 2 gene expression in human colorectal adenomas and adenocarcinomas. Gastroenterology 107: 1183-1188, 1994.

31. Eltze E, Wülfing C, Von Struensee D, Piechota H, Buerger H and Hertle L: Cox-2 and Her2/neu co-expression in invasive bladder cancer. Int J Oncol 26: 1525-1531, 2005.

32. Ristimäki A, Sivula A, Lundin J, Lundin M, Salminen T, Haglund $\mathrm{C}$, Joensuu $\mathrm{H}$ and Isola J: Prognostic significance of elevated cyclooxygenase- 2 expression in breast cancer. Cancer Res 62: 632-635, 2002.

33. Larkins TL, Nowell M, Singh S and Sanford GL: Inhibition of cyclooxygenase-2 decreases breast cancer cell motility, invasion and matrix metalloproteinase expression. BMC Cancer 6: 181-192, 2006.

34. Davies G, Salter J, Hills M, Martin LA, Sacks N and Dowsett M: Correlation between cyclooxygenase-2 expression and angiogenesis in human breast cancer. Clin Cancer Res 9: 2651-2656, 2003.

35. Costa C, Soares R, Reis-Filho JS, Leitão D, Amendoeira I and Schmitt FC: Cyclo-oxygenase 2 expression is associated with angiogenesis and lymph node metastasis in human breast cancer. J Clin Pathol 55: 429-434, 2002.

36. Mehner C,Hockla A, MillerE, Ran S, Radisky DC and Radisky ES: Tumor cell-produced matrix metalloproteinase 9 (MMP-9) drives malignant progression and metastasis of basal-like triple negative breast cancer. Oncotarget 5: 2736-2749, 2014.

37. Scorilas A, Karameris A, Arnogiannaki N, Ardavanis A, Bassilopoulos P, Trangas T and Talieri M: Overexpression of matrix-metalloproteinase-9 in human breast cancer: A potential favourable indicator in node-negative patients. Br J Cancer 84: 1488-1496, 2001

38. Itoh T, Tanioka M, Yoshida H, Yoshioka T, Nishimoto $\mathrm{H}$ and Itohara S: Reduced angiogenesis and tumor progression in gelatinase A-deficient mice. Cancer Res 58: 1048-1051, 1998.

39. Coussens LM, Tinkle CL, Hanahan D and Werb Z: MMP-9 supplied by bone marrow-derived cells contributes to skin carcinogenesis. Cell 103: 481-490, 2000.

40. Huang S, Day TW, Choi MR and Safa AR: Human beta-galactoside alpha-2,3-sialyltransferase (ST3Gal III) attenuated Taxol-induced apoptosis in ovarian cancer cells by downregulating caspase-8 activity. Mol Cell Biochem 331: 81-88, 2009. 